\title{
ON SUBORDINATE RANDOM WALKS
}

\author{
ANTE MIMICA
}

\begin{abstract}
In this article subordination of random walks in $\mathbb{R}^{d}$ is considered. We prove that subordination of random walks in the sense of [BSC12] yields the same process as subordination of Lévy processes (in the sense of Bochner). Furthermore, we prove that appropriately scaled subordinate random walk converges to a multiple of a rotationally $2 \alpha$-stable process if and only if the Laplace exponent of the corresponding subordinator varies regularly at zero with index $\alpha \in(0,1]$.
\end{abstract}

\section{INTRODUCTION}

A subordinator $T=\left(T_{t}\right)_{t \geqslant 0}$ is a non-decreasing Lévy process (i.e. a stochastic process having stationary and independent increments) defined on a probability space $(\Omega, \mathcal{F}, \mathbb{P})$. From the definition it follows that $T$ takes values in $[0, \infty)$ and the Laplace transform of $T_{t}$ is given by

$$
\mathbb{E} e^{-\lambda T_{t}}=e^{-t \phi(\lambda)}, \quad \lambda>0
$$

where $\phi$ is called the Laplace exponent of $T$. It is of the following form (see [Ber96, III.1])

$$
\phi(\lambda)=b \lambda+\int_{(0, \infty)}\left(1-e^{-\lambda s}\right) \mu(d s)
$$

where $b \geqslant 0$ is the drift of the subordinator and $\mu$ is a measure on $(0, \infty)$ satisfying $\int_{(0, \infty)}(1 \wedge s) \mu(d s)<\infty$ called the Lévy measure of $T$. It is known that $\phi$ is a Bernstein function, meaning that $\phi:(0, \infty) \rightarrow \mathbb{R}$ is a $C^{\infty}$-function satisfying

$$
\phi(\lambda) \geqslant 0 \text { for all } \quad \lambda>0
$$

and

$$
(-1)^{n-1} \phi^{(n)}(\lambda) \geqslant 0 \quad \text { for all } \quad \lambda>0 \text { and } n \in \mathbb{N}
$$

(here $\phi^{(n)}$ denotes the $n$-th derivative of $\phi$ ). It is known that every Bernstein function $\phi$ satisfying $\lim _{\lambda \downarrow 0} \phi(\lambda)=0$ has a unique representation given by (1.1) (see [SSV12, Theorem $3.2])$.

Let $Z=\left(Z_{n}\right)_{n \geqslant 0}$ be a random walk in $\mathbb{R}^{d}$ independent of $T$, that is $Z_{0}=0$ and $Z_{n}=\zeta_{1}+\ldots+\zeta_{n}$ for $n \geqslant 1$, where $\left(\zeta_{n}\right)_{n \geqslant 1}$ is a sequence of independent and identically distributed random vectors in $\mathbb{R}^{d}$ defined on the probability space $(\Omega, \mathcal{F}, \mathbb{P})$ idependent of $T$.

2010 Mathematics Subject Classification. Primary 60J75, Secondary 60G51, 60G52, 60F17.

Key words and phrases. random walk, subordination, compound Poisson process, Lévy process, regular variation, invariance principle.

Research supported by Croatian Science Foundation under the project 3526. 
We define subordinate random walk $X=\left(X_{n}\right)_{n \geqslant 0}$ as

$$
X_{0}=0 \quad \text { and } \quad X_{n}=\sum_{k=1}^{n} \xi_{k} \text { for } n \geqslant 1,
$$

where $\xi_{1}, \xi_{2}, \ldots$ are independent and identically distributed having the following distribution

$$
\mathbb{P}\left(\xi_{1} \in B\right)=b \mathbb{P}\left(Z_{1} \in B\right)+\sum_{m=1}^{\infty} \int_{(0, \infty)} \frac{q^{m-1} t^{m}}{m !} e^{-q t} \mu(d t) \mathbb{P}\left(Z_{m} \in B\right), \quad B \subset \mathbb{R}^{d} \text { Borel }
$$

and $q>0$ is such that $\phi(q)=q$.

Remark 1.1. The choice of $q>0$ is made so that $\mathbb{P}\left(\xi_{1} \in \mathbb{R}^{d}\right)=1$ :

$$
\mathbb{P}\left(\xi_{1} \in \mathbb{R}^{d}\right)=b+q^{-1} \int_{(0, \infty)}\left(e^{q t}-1\right) e^{-q t} \mu(d t)=\frac{\phi(q)}{q}=1 .
$$

The condition $\phi(q)=q$ is not a big restriction, since we can always consider normalization $\psi(\lambda)=q \phi(q)^{-1} \phi(\lambda)$ of $\phi$ that satisfies the latter condition.

This procedure is known as discrete subordination introduced in [BSC12]. In this article we will embed subordinate random walks into a Lévy process (actually compound Poisson processes) by using time change by a Poisson process (see [Sat99, Section 1.4] and Section 2). This gives us an opportunity to link discrete subordination with subordination of Lévy processes (in the sense of Bochner, see [SSV12, Chapter 13]). It turns out that, understood as Lévy processes just described, discrete subordination and subordination of the random walk by the subordinator give rise to the same process as the following proposition states.

Proposition 1.2. Let $Z=\left(Z_{n}\right)_{n \geqslant 0}$ be a random walk in $\mathbb{R}^{d}, T=\left(T_{t}\right)_{t \geqslant 0}$ an independent nonconstant subordinator and let $X=\left(X_{n}\right)_{n \geqslant 0}$ be the corresponding discrete subordinate random walk defined by (1.2) and (1.3). If $N=\left(N_{t}\right)_{t \geqslant 0}$ is a Poisson process with intensity $q>0$ independent of $X, T$ and $Z$, then the processes $\hat{X}=\left(\hat{X}_{t}\right)_{t \geqslant 0}$ and $\tilde{X}=\left(\tilde{X}_{t}\right)_{t \geqslant 0}$ defined by

$$
\hat{X}_{t}=X_{N_{t}} \quad \text { and } \quad \tilde{X}_{t}=Z_{N_{T_{t}}}, \quad t \geqslant 0
$$

are compound Poisson processes having the same charcteristics.

Let $Z=\left(Z_{n}\right)_{n \geqslant 0}$ be the simple symmetric random walk in $\mathbb{Z}^{d}$, that is $Z_{0}=0$, $Z_{n}=\zeta_{1}+\ldots+\zeta_{n}$, for $n \geqslant 1$ and $\left(\zeta_{n}\right)_{n \geqslant 1}$ is a sequence of independent and identically distributed random vectors in $\mathbb{Z}^{d}$ such that $\mathbb{P}\left(\zeta_{1}=e_{j}\right)=\mathbb{P}\left(\zeta_{1}=-e_{j}\right)=\frac{1}{2 d}$, where $e_{j}=(0, \ldots, 0,1,0, \ldots, 0) \in \mathbb{Z}^{d}$ has 1 at the $j$-th coordinate, $1 \leqslant j \leqslant d$.

Consider a nonconstant subordinator $T=\left(T_{t}\right)_{t \geqslant 0}$ with the Laplace exponent $\phi$ and a sequence $\left(\xi_{n}\right)_{n \geqslant 1}$ of independent and identically distributed random variables with the distribution given by (1.3) with $q=1$. Note that $\phi$ strictly increases (so that the inverse $\phi^{-1}$ exists). We aim to consider convergence of the processes $X^{(n)}=\left(X_{t}^{(n)}\right)_{t \geqslant 0}, n \in \mathbb{N}$ defined by

$$
X_{t}^{(n)}=\sqrt{\phi^{-1}\left(n^{-1}\right)} \sum_{k=1}^{N_{n t}} \xi_{k}, \quad n \geqslant 0,
$$


where $N=\left(N_{t}\right)_{t \geqslant 0}$ is a stochastic process that is either a Poisson proces with intensity 1 independent of the sequence $\left(\xi_{n}\right)_{n \geqslant 1}$ or $N_{t}=\lfloor t\rfloor, t \geqslant 0$. In the former case (see Proposition $1.2)$

$$
X_{t}^{(n)} \stackrel{d}{=} \sqrt{\phi^{-1}\left(n^{-1}\right)} Z_{N_{T_{n t}}}
$$

while in the latter case

$$
X_{t}^{(n)}=\sqrt{\phi^{-1}\left(n^{-1}\right)} \sum_{k=1}^{\lfloor n t\rfloor} \xi_{k} .
$$

Our aim is to investigate (weak) limits of the processes $X^{(n)}$, i.e. we will establish a type of a functional limit theorem by imposing conditions on the Laplace exponent $\phi$. Note that the processes defined by (1.5) are actually random sums and find their application in economics, financial mathematics and queuing theory(see [EKM03],[Gut09]).

The following tightness result in the space

$$
D\left([0, \infty), \mathbb{R}^{d}\right):=\left\{f:[0, \infty) \rightarrow \mathbb{R}^{d}: f \text { is right continuous with left limits }\right\}
$$

(with an appropriate topology) will be proved in Section 3. Condition that will ensure tightness for subordinate random walk is certain upper scaling condition of the inverse of the inverse of the Laplace exponent.

Proposition 1.3. Assume that the the Laplace exponent $\phi$ of a subordinator satisfies $\phi(1)=1$ and there exist $c>0$ and $\gamma>1$ such that $\limsup _{\lambda \downarrow 0} \frac{\phi^{-1}(\lambda x)}{\phi^{-1}(\lambda)} \leqslant c x^{\gamma}$ for all $x \geqslant 1$.

Then the sequence $\left(X^{(n)}\right)_{n \geqslant 1}$ defined by (1.4) is tight in the space $D\left([0, \infty), \mathbb{R}^{d}\right)$.

Now we explore convergence of the sequence $\left(X^{(n)}\right)_{n \geqslant 1}$. A measurable function $f:(0, \infty) \rightarrow$ $(0, \infty)$ is said to vary regularly at 0 with index $\rho \in \mathbb{R}$ if

$$
\lim _{\lambda \downarrow 0} \frac{f(\lambda x)}{f(\lambda)}=x^{\rho} \quad \text { for all } \quad x>0 .
$$

A Lévy process (see Section 2) $W^{(\alpha)}=\left(W_{t}^{(\beta)}\right)_{t \geqslant 0}$ is called rotationally invariant $\beta$-stable process in $\mathbb{R}^{d}(\beta \in(0,2])$ if

$$
\mathbb{E}\left[e^{i \vartheta \cdot W_{t}^{(\beta)}}\right]=e^{-t|\vartheta|^{\beta}} \quad \text { for all } \quad \vartheta \in \mathbb{R}^{d} \quad \text { and } \quad t \geqslant 0 .
$$

Theorem 1.4. Assume that the Laplace exponent $\phi$ of a subordinator satisfies $\phi(1)=1$. The sequence $\left(X^{(n)}\right)_{n \geqslant 1}$ converges in $D\left([0, \infty), \mathbb{R}^{d}\right)$ if and only if $\phi$ varies regularly at zero with index $\alpha \in(0,1]$. In this case the limit is $(2 d)^{-\alpha} W^{(2 \alpha)}$.

The following example is interesting, since it approximates Brownian motion by a compound Poisson process that has steps with infinite variance.

Example 1.5. Consider a Bernstein function

$$
\phi(\lambda)=c \lambda \log \left(1+\frac{1}{\lambda}\right), \quad c=\frac{1}{\log 2} .
$$


Since $\lim _{\lambda \downarrow 0} \phi(\lambda)=0, \phi$ is the Laplace exponent of a subordinator. Note that $\phi$ varies regularly at 0 with index 1 . By Theorem 1.4, the corresponding subordinate random walk

$$
X_{t}^{(n)}=\sqrt{\phi^{-1}\left(n^{-1}\right)} \sum_{i=1}^{\lfloor n t\rfloor} \xi_{i}
$$

converges to a multiple of Brownian motion by $(2 d)^{-1}$. Let $d=1$. Unlike in Donsker's invariance principle, $\mathbb{E} \xi_{1}^{2}=+\infty$. Indeed,

$$
\begin{aligned}
\mathbb{E} \xi_{1}^{2} & =\sum_{m=1}^{\infty} \mathbb{E} Z_{m}^{2} \int_{(0, \infty)} \frac{t^{m}}{m !} e^{-t} \mu(d t)=\sum_{m=1}^{\infty} m \int_{(0, \infty)} \frac{t^{m}}{m !} e^{-t} \mu(d t) \\
& =\int_{(0, \infty)} \sum_{m=1}^{\infty} \frac{t^{m}}{(m-1) !} e^{-t} \mu(d t)=\int_{(0, \infty)} t \mu(d t)=\int_{0}^{\infty} \mu(t, \infty) d t .
\end{aligned}
$$

By [SSV12, pp. 230-231, table entry 27], the Lévy measure of $\phi$ is given by

$$
\mu(d t)=c \frac{1-e^{-t}(1+t)}{t^{2}} d t
$$

and so

$$
\lim _{t \rightarrow \infty} \frac{\mu(t, \infty)}{\frac{1}{t}}=c .
$$

Therefore, there exists a constant $c_{1}>0$ so that

$$
\mathbb{E} \xi_{1}^{2} \geqslant c_{1} \int_{1}^{\infty} \frac{d t}{t}=+\infty
$$

\section{LÉVY PROCESSES}

A stochastic process $X=\left(X_{t}\right)_{t \geqslant 0}$ defined on a probability space $(\Omega, \mathcal{F}, \mathbb{P})$ taking values in $\mathbb{R}^{d}(d \geqslant 1)$ is a Lévy process if $X_{0}=0$, it has stationary and independent incremets and paths that are $\mathbb{P}$-a.s. right-continuous with left limits. It is well known that the characteristic function of $X_{t}$ is

$$
\mathbb{E} e^{i \vartheta \cdot X_{t}}=e^{-t \psi(\vartheta)}, \quad t>0, \vartheta \in \mathbb{R}^{d},
$$

where $\psi: \mathbb{R}^{d} \rightarrow \mathbb{C}$ is called the characteristic exponent of $X$ and has the following LévyKhintchine representation

$$
\psi(\vartheta)=i \beta \cdot \vartheta+\frac{1}{2} Q \vartheta \cdot \vartheta+\int_{\mathbb{R}^{d} \backslash\{0\}}\left(1-e^{i y \cdot \vartheta}+i y \cdot \vartheta 1_{\{|y| \leqslant 1\}}\right) \nu(d y), \quad \vartheta \in \mathbb{R}^{d} .
$$

Here $\beta=\left(\beta_{1}, \ldots, \beta_{d}\right) \in \mathbb{R}^{d}, Q$ is a $d \times d$ positive semi-definite matrix and $\nu$ is the Lévy measure, i.e. a measure on $\mathbb{R}^{d} \backslash\{0\}$ satisfying $\int_{\mathbb{R}^{d} \backslash\{0\}}\left(1 \wedge|y|^{2}\right) \nu(d y)<\infty$. If $\beta=0$ and $Q=0$, we will call $X$ a pure jump Lévy process. A triplet $(\beta, Q, \nu)$ is called the Lévy triplet of $X$ and every Lévy process is uniquely determined by its Lévy triplet (see [Sat99, Section 3.11]). 
A particular example of a Lévy process that will be used in this section is a compound Poisson process (see [Sat99, Section 1.4]). It is a Lévy process $X=\left(X_{t}\right)_{t \geqslant 0}$ with the characteristic exponent of the form

$$
\psi(\vartheta)=q \int_{\mathbb{R}^{d}}\left(1-e^{i y \cdot \vartheta}\right) \eta(d y), \quad \vartheta \in \mathbb{R}^{d},
$$

where $q>0$ and $\eta$ is a measure on $\mathbb{R}^{d}$ such that $\eta(\{0\})=0$. The corresponding Lévy triplet is $(\beta, 0, \nu)$, where

$$
\beta=\left(\beta_{1}, \ldots, \beta_{d}\right), \beta_{j}=-q \int_{0<|y| \leqslant 1} y_{j} \eta(d y), j=1, \ldots, d
$$

and

$$
\nu(B)=q \eta(B), \quad B \subset \mathbb{R}^{d} \text { Borel } .
$$

In particular, for $\eta=\delta_{1}, X$ is the Poisson process with intensity $q$.

The following construction of a compound Poisson process will be useful (see [Sat99, Theorem 1.4.2]). Let $N=\left(N_{t}\right)_{t \geqslant 0}$ be the Poisson process with intensity $q>0$ and let $\left(\zeta_{n}\right)_{n \in \mathbb{N}}$ be a sequence of independent random variables with law $\rho$ defined on a common probability space $(\Omega, \mathcal{F}, \mathbb{P})$ independent of $N$. Define a random walk $Z=\left(Z_{n}\right)_{n \geqslant 0}$ by $Z_{0}=0$ and $Z_{n}=\zeta_{1}+\ldots+\zeta_{n}$ for $n \geqslant 1$. Then the process $X=\left(X_{t}\right)_{t \geqslant 0}$ defined by $X_{t}=Z_{N_{t}}$ is a compound Poisson process.

Proof of Proposition 1.2. The process $\tilde{Z}=\left(\tilde{Z}_{t}\right)_{t \geqslant 0}$ defined by $\tilde{Z}_{t}=Z_{N_{t}}$ is a compound Poisson process with (see (2.2) and (2.3))

$$
\beta_{\tilde{Z}}=-q \int_{0<|y| \leqslant 1} y \mathbb{P}\left(\zeta_{1} \in d y\right) \quad \text { and } \quad \nu_{\tilde{Z}}(d y)=q \mathbb{P}\left(\zeta_{1} \in d y\right) .
$$

By [Sat99, Theorem 30.1], $\tilde{X}$ is a Lévy process with the Lévy triplet $\left(\beta_{\tilde{X}}, 0, \nu_{\tilde{X}}\right)$, where

$$
\beta_{\tilde{X}}=b \beta_{\tilde{Z}}-\int_{(0, \infty)} \int_{0<|y| \leqslant 1} y \mathbb{P}\left(\tilde{Z}_{s} \in d y\right) \mu(d s)
$$

and

$$
\nu_{\tilde{X}}(B)=q b \mathbb{P}\left(\zeta_{1} \in B\right)+\int_{(0, \infty)} \mathbb{P}\left(\tilde{Z}_{s} \in B\right) \mu(d s) .
$$

On the other hand, $\hat{X}$ is a compound Poisson process and its Lévy triplet $\left(\beta_{\hat{X}}, 0, \nu_{\hat{X}}\right)$ is, by $(2.2)$ and (2.3), given by

$$
\beta_{\hat{X}}=-q \int_{0<|y| \leqslant 1} y \mathbb{P}\left(\xi_{1} \in d y\right) \quad \text { and } \quad \nu_{\hat{X}}(d y)=q \mathbb{P}\left(\xi_{1} \in d y\right) .
$$

Then (1.3) yields

$$
\begin{aligned}
\beta_{\hat{X}} & =-q b \int_{0<|y| \leqslant 1} y \mathbb{P}\left(Z_{1} \in d y\right)-\int_{(0, \infty)} \int_{0<|y| \leqslant 1} \sum_{m=1}^{\infty} \frac{(q s)^{m}}{m !} e^{-q s} y \mathbb{P}\left(Z_{m} \in d y\right) \mu(d s) \\
& =-q b \int_{0<|y| \leqslant 1} y \mathbb{P}\left(\zeta_{1} \in d y\right)-\int_{(0, \infty)} \int_{0<|y| \leqslant 1} y \mathbb{P}\left(Z_{N_{s}} \in d y\right) \mu(d s)=\beta_{\tilde{X}}
\end{aligned}
$$


and, for a Borel set $B \subset \mathbb{R}^{d} \backslash\{0\}$,

$$
\begin{aligned}
\nu_{\hat{X}}(B) & =q b \mathbb{P}\left(Z_{1} \in B\right)+\int_{(0, \infty)} \sum_{m=1}^{\infty} \frac{(q s)^{m}}{m !} e^{-q s} \mathbb{P}\left(Z_{m} \in B\right) \mu(d s) \\
& =q b \mathbb{P}\left(\zeta_{1} \in B\right)+\int_{(0, \infty)} \mathbb{P}\left(Z_{N_{s}} \in B\right) \mu(d s)=\nu_{\tilde{X}}(B) .
\end{aligned}
$$

Hence, $\hat{X}$ and $\tilde{X}$ are Lévy processes with same Lévy triplets.

\section{Tightness Result}

We recall that the process $X^{(n)}$ was defined by

$$
X_{t}^{(n)}=\sqrt{\phi^{-1}\left(n^{-1}\right)} \sum_{k=0}^{N_{n t}} \xi_{k}, \quad n \geqslant 0,
$$

where $T=\left(T_{t}\right)_{t \geqslant 0}$ is a subordinator with the Laplace exponent $\phi,\left(\xi_{n}\right)_{n \geqslant 1}$ is a sequence of independent and identically distributed random vectors with the distribution given by (1.3) and $N=\left(N_{t}\right)_{t \geqslant 0}$ is either the Poisson process with intensity 1 independent of the sequence $\left(\xi_{n}\right)_{n \geqslant 1}$ or $N_{t}=\lfloor t\rfloor$ for $t \geqslant 0$. The aim of this section is to prove that the sequence $\left(X^{(n)}\right)_{n \geqslant 1}$ is tight in the Skorokhod space $D\left([0, \infty), \mathbb{R}^{d}\right)$ endowed with the Skorokhod topology. We refer the reader to [JS03, VI.1b] for a definition of the Skorokhod topology.

Lemma 3.1. Let $\phi:(0, \infty) \rightarrow(0, \infty)$ be a the Laplace exponent of a subordinator with the Lévy measure $\mu$.

(i) Then

$$
\int_{(0, r]} t \mu(d t) \leqslant \operatorname{er\phi }\left(r^{-1}\right) \quad \text { for all } \quad r>0
$$

and

$$
\mu(t, \infty) \leqslant\left(1-e^{-1}\right)^{-1} \phi\left(t^{-1}\right) \quad \text { for all } \quad t>0 .
$$

(ii) For all $\lambda, x>0$ we have $\phi(\lambda x) \leqslant(x \vee 1) \phi(\lambda)$.

(iii) If $\phi$ is trictly increasing and varies regularly at 0 with index $\alpha>0$, then $\phi^{-1}$ varies regularly at 0 with index $1 / \alpha$.

Remark 3.2. Lemma 3.1 (iii) can be compared with [BGT87, Proposition 1.5.15], where asymptotic inverse and conjugacy are considered. Although this is a special case of the aforementioned result, we give simplified proof for strictly increasing functions $\phi$.

Proof. (i) Starting from (1.1) and using an elementary inequality

$$
1-e^{-x} \geqslant x e^{-x}, x \geqslant 0
$$

it follows that

$$
\phi(\lambda) \geqslant \int_{\left(0, \lambda^{-1}\right]}\left(1-e^{-\lambda t}\right) \mu(d t) \geqslant \int_{\left(0, \lambda^{-1}\right]} \lambda t e^{-\lambda t} \mu(d t) \geqslant e^{-1} \lambda \int_{\left(0, \lambda^{-1}\right]} t \mu(d t),
$$


yielding the first estimate. By (1.1),

$$
\phi(\lambda) \geqslant \int_{\left(\lambda^{-1}, \infty\right)}\left(1-e^{-\lambda t}\right) \mu(d t) \geqslant\left(1-e^{-1}\right) \mu\left(\lambda^{-1}, \infty\right)
$$

and the second estimate follows.

(ii) Let $\lambda>0$. If $x \geqslant 1$, then $1-e^{-t x} \leqslant x\left(1-e^{-t}\right)$ for all $t \geqslant 0$, hence

$$
\phi(\lambda x)=b \lambda x+\int_{(0, \infty)}\left(1-e^{-\lambda x t}\right) \mu(d t) \leqslant b \lambda x+\int_{(0, \infty)} x\left(1-e^{-\lambda t}\right) \mu(d t)=x \phi(\lambda) .
$$

For $x \leqslant 1$ we use that $\phi$ is non-decreasing to conclude that $\phi(\lambda x) \leqslant \phi(\lambda)$.

(iii) Let $x>0$. By regular variation of $\phi$, for any $0<\varepsilon<x^{\alpha}$ there exists $\delta>0$ such that

$$
x^{\alpha}-\varepsilon \leqslant \frac{\phi(\lambda x)}{\phi(\lambda)} \leqslant x^{\alpha}+\varepsilon, 0<\lambda<\delta .
$$

Since $\phi$ is strictly increasing and continuous, the last display implies

$$
\limsup _{\lambda \downarrow 0} \frac{\phi^{-1}\left(\lambda\left(x^{\alpha}-\varepsilon\right)\right)}{\phi^{-1}(\lambda)} \leqslant x \leqslant \liminf _{\lambda \downarrow 0} \frac{\phi^{-1}\left(\lambda\left(x^{\alpha}+\varepsilon\right)\right)}{\phi^{-1}(\lambda)} .
$$

Let $y>0$. By taking $x=(y+\varepsilon)^{1 / \alpha}$ we get $\limsup _{\lambda \downarrow 0} \frac{\phi^{-1}(\lambda y)}{\phi^{-1}(\lambda)} \leqslant(y+\varepsilon)^{1 / \alpha}$ and, since $\varepsilon>0$ was arbitrary, this implies $\limsup _{\lambda \downarrow 0} \frac{\phi^{-1}(\lambda y)}{\phi^{-1}(\lambda)} \leqslant y^{1 / \alpha}$. For $0<\varepsilon<y$ and $x=(y-\varepsilon)^{1 / \alpha}$ we obtain $\liminf _{\lambda \downarrow 0} \frac{\phi^{-1}(\lambda y)}{\phi^{-1}(\lambda)} \geqslant(y-\varepsilon)^{1 / \alpha}$ and so $\liminf _{\lambda \downarrow 0} \frac{\phi^{-1}(\lambda y)}{\phi^{-1}(\lambda)} \geqslant y^{1 / \alpha}$.

Lemma 3.3. Assume that $\phi$ is strictly increasing with $b=0$ and $\phi(1)=1$. There exists a constant $c_{0}>0$ such that for any $K, \beta, a>0$ and $n \in \mathbb{N}$ we have

$$
\mathbb{P}\left(\left|\sum_{k \leqslant a n} \xi_{k}\right|>\frac{K}{\sqrt{\phi^{-1}\left(n^{-1}\right)}}\right) \leqslant c_{0} a\left(K^{-2-\beta} \frac{\left.\phi^{-1}\left(n^{-1}\right)\right)}{\phi^{-1}\left(K^{-\beta} n^{-1}\right)}+K^{-\beta}\right) .
$$

Proof. Let $\left\{\left(Z_{n}^{(k)}\right)_{n \geqslant 1}: k \in \mathbb{N}\right\}$ be a family of independent copies of the random walk $\left(Z_{n}\right)_{n \geqslant 1}$ defined by $Z_{0}=0, Z_{n}=\zeta_{1}+\ldots+\zeta_{n}$, for $n \geqslant 1$, where $\left(\zeta_{n}\right)_{n \geqslant 1}$ is a sequence of independent and identically distributed random vectors in $\mathbb{Z}^{d}$ such that $\mathbb{P}\left(\zeta_{1}=e_{j}\right)=$ $\mathbb{P}\left(\zeta_{1}=-e_{j}\right)=\frac{1}{2 d}, j=1, \ldots, d$. It follows from Chebyshev inequality that, for $r>0$ and $l, m_{1}, \ldots, m_{l} \in \mathbb{N}$ the following holds

$$
\mathbb{P}\left(\left|\sum_{k=1}^{l} Z_{m_{k}}^{(k)}\right|>r\right) \leqslant \frac{\mathbb{E}\left|\sum_{k=1}^{l} Z_{m_{k}}^{(k)}\right|^{2}}{r^{2}}=\frac{m_{1}+\ldots+m_{l}}{r^{2}}
$$

and this gives the following estimate

$$
\mathbb{P}\left(\left|\sum_{k=1}^{l} Z_{m_{k}}^{(k)}\right|>r\right) \leqslant \frac{m_{1}}{r^{2}} \wedge 1+\ldots+\frac{m_{l}}{r^{2}} \wedge 1 .
$$


By (1.3) with $q=1$ and (3.1),

$$
\begin{aligned}
\mathbb{P}\left(\left|\sum_{k \leqslant a n} \xi_{k}\right|>\right. & \left.\frac{K}{\sqrt{\phi^{-1}\left(n^{-1}\right)}}\right) \\
& =\sum_{m_{1}, \ldots, m_{\lfloor a n\rfloor}=1}^{\infty} \prod_{j \leqslant a n} \int_{(0, \infty)} \frac{t^{m_{j}}}{m_{j} !} e^{-t} \mu(d t) \mathbb{P}\left(\left|\sum_{k \leqslant a n} Z_{m_{k}}^{(k)}\right|>\frac{K}{\sqrt{\phi^{-1}\left(n^{-1}\right)}}\right) \\
& \leqslant \sum_{i \leqslant a n} \sum_{m_{i}=1}^{\infty} \int_{(0, \infty)} \frac{t^{m_{i}}}{m_{i} !} e^{-t} \mu(d t)\left(\frac{m_{i} \phi^{-1}\left(n^{-1}\right)}{K^{2}} \wedge 1\right) \prod_{\substack{j \leqslant a n \\
j \neq i}}\left(\sum_{m_{j}=1}^{\infty} \int_{(0, \infty)} \frac{t^{m_{j}}}{m_{j} !} e^{-t} \mu(d t)\right) .
\end{aligned}
$$

Since $\sum_{m=1}^{\infty} \int_{(0, \infty)} \frac{t^{m}}{m !} e^{-t} \mu(d t)=\int_{(0, \infty)}\left(e^{t}-1\right) e^{-t} \mu(d t)=\phi(1)=1$, the product term in the last display is equal to 1 and so, for $\beta>0$,

$$
\begin{aligned}
\mathbb{P}\left(\left|\sum_{k \leqslant a n} \xi_{k}\right|>\frac{K}{\sqrt{\phi^{-1}\left(n^{-1}\right)}}\right) \leqslant a n \int_{(0, \infty)} \sum_{m=1}^{\infty} \frac{t^{m}}{m !}\left(\frac{m_{i} \phi^{-1}\left(n^{-1}\right)}{K^{2}} \wedge 1\right) \mu(d t) \\
\leqslant \operatorname{an}\left(\int_{\left(0, \phi^{-1}\left(K^{-\beta} n^{-1}\right)^{-1}\right]} t \mu(d t) K^{-2} \phi^{-1}\left(n^{-1}\right)+\int_{\left(\phi^{-1}\left(K^{-\beta} n^{-1}\right)^{-1}, \infty\right)} \mu(d t)\right) .
\end{aligned}
$$

Now we apply Lemma 3.1 (i) to get

$$
\begin{aligned}
\mathbb{P}\left(\left|\sum_{k \leqslant a n} \xi_{k}\right|\right. & \left.>\frac{K}{\sqrt{\phi^{-1}\left(n^{-1}\right)}}\right) \\
& \leqslant \operatorname{an}\left(e \phi^{-1}\left(K^{-\beta} n^{-1}\right)^{-1} K^{-\beta} n^{-1} K^{-2} \phi^{-1}\left(n^{-1}\right)+\frac{e}{e-1} K^{-\beta} n^{-1}\right) \\
& =a e K^{-2-\beta} \frac{\phi^{-1}\left(n^{-1}\right)}{\phi^{-1}\left(K^{-\beta} n^{-1}\right)}+\frac{a e}{e-1} K^{-\beta} .
\end{aligned}
$$

Recall that a random time $\tau$ is a stopping time with respect to the process $X^{(n)}$ if $\{\tau \leqslant t\} \in \sigma\left(X_{s}^{(n)}: s \leqslant t\right)$ for any $t>0$. In particular, if $N_{t}=\lfloor t\rfloor$, then $\sigma\left(X_{s}^{(n)}: s \leqslant t\right)=$ $\sigma\left(\xi_{k}: k \leqslant n t\right)$ and so

$$
\{\lfloor n \tau\rfloor=m\}=\left\{\frac{m}{n} \leqslant \tau<\frac{m+1}{n}\right\} \in \sigma\left(\xi_{k}: k \leqslant m\right), m \in \mathbb{N} \cup\{0\} .
$$

Lemma 3.4. Let $\left(\tau_{n}\right)_{n \geqslant 1}$ be a sequence of random times such that $\tau_{n}$ is a stopping time for the process $X^{(n)}$ for any $n \in \mathbb{N}$ and let $\left(h_{n}\right)$ be a sequence of non-negative numbers. There exists a sequence of functions $g_{n}: \mathbb{R}^{d} \rightarrow[0, \infty)$ satisfying $\lim _{n \rightarrow \infty} g_{n}(\vartheta)=1$ for all $\vartheta \in \mathbb{R}^{d}$ such that

$$
E\left[e^{i \vartheta \cdot\left(X_{\tau_{n}+h_{n}}^{(n)}-X_{\tau_{n}}^{(n)}\right)}\right]=\mathbb{E}\left[e^{i \vartheta \cdot X_{h_{n}}^{(n)}}\right] g_{n}(\vartheta), \quad \vartheta \in \mathbb{R}^{d}
$$

Proof. If $N$ is a Poisson process, then $X^{(n)}$ is a Lévy process and so the claim follows from the strong Markov property for Lévy processes with $g_{n} \equiv 1, n \in \mathbb{N}$.

Let us consider now the case $N_{t}=\lfloor t\rfloor$. First we remark that

$$
Z_{n}:=N_{\tau_{n}+h_{n}}-N_{\tau_{n}}-N_{h_{n}}=\left\lfloor n\left(\tau_{n}+h_{n}\right)\right\rfloor-\left\lfloor n \tau_{n}\right\rfloor-\left\lfloor n h_{n}\right\rfloor \in\{0,1\} \mathbb{P}-\text { a.s., } n \in \mathbb{N} \text {. }
$$


For $\vartheta \in \mathbb{R}^{d}$ we calculate

$$
\begin{aligned}
\mathbb{E} & {\left[e^{i \vartheta \cdot\left(X_{\tau_{n}+h_{n}}^{(n)}-X_{\tau_{n}}^{(n)}\right)}\right] } \\
& =\mathbb{E}\left[\exp \left\{i \sqrt{\phi^{-1}\left(n^{-1}\right)} \sum_{k=\left\lfloor n \tau_{n}\right\rfloor+1}^{\left\lfloor n \tau_{n}\right\rfloor+\left\lfloor n h_{n}\right\rfloor} \vartheta \cdot \xi_{k}\right\} e^{i \sqrt{\left.\phi^{-1}\left(n^{-1}\right)\right)}} Z_{n} \vartheta \cdot \xi_{\left\lfloor n \tau_{n}\right\rfloor+\left\lfloor n h_{n}\right\rfloor+1}\right] \\
& =\sum_{m=0}^{\infty} \mathbb{E}\left[\exp \left\{i \sqrt{\phi^{-1}\left(n^{-1}\right)} \sum_{k=m+1}^{m+\left\lfloor n h_{n}\right\rfloor} \vartheta \cdot \xi_{k}\right\} e^{i \sqrt{\left.\phi^{-1}\left(n^{-1}\right)\right)}} Z_{n} \vartheta \cdot \xi_{m+\left\lfloor n h_{n}\right\rfloor+1}\left\lfloor\left\lfloor n \tau_{n}\right\rfloor=m\right] .\right.
\end{aligned}
$$

Now we use that $\left(\xi_{k}\right)_{k \geqslant 1}$ are independent and identically distributed and (3.2) to deduce

$$
\begin{aligned}
\mathbb{E} & {\left[e^{i \vartheta \cdot\left(X_{\tau_{n}+h_{n}}^{(n)}-X_{\tau_{n}}^{(n)}\right)}\right] } \\
& =\sum_{m=0}^{\infty} \mathbb{E}\left[\exp \left\{i \sqrt{\phi^{-1}\left(n^{-1}\right)} \sum_{k=m+1}^{m+\left\lfloor n h_{n}\right\rfloor} \vartheta \cdot \xi_{k}\right\}\right] \mathbb{E}\left[e^{i \sqrt{\left.\phi^{-1}\left(n^{-1}\right)\right)} Z_{n} \vartheta \cdot \xi_{m+\left\lfloor n h_{n}\right\rfloor+1}}\right] \mathbb{P}\left(\left\lfloor n \tau_{n}\right\rfloor=m\right) \\
& =\mathbb{E}\left[\exp \left\{i \sqrt{\phi^{-1}\left(n^{-1}\right)} \sum_{k=1}^{\left\lfloor n h_{n}\right\rfloor} \vartheta \cdot \xi_{k}\right\}\right] \mathbb{E}\left[e^{i \sqrt{\left.\phi^{-1}\left(n^{-1}\right)\right)} Z_{n} \vartheta \cdot \xi_{1}}\right] .
\end{aligned}
$$

By dominated convergence theorem,

$$
g_{n}(\vartheta):=\mathbb{E}\left[e^{i \sqrt{\left.\phi^{-1}\left(n^{-1}\right)\right)} Z_{n} \vartheta \cdot \xi_{1}}\right], \vartheta \in \mathbb{R}^{d}, n \in \mathbb{N}
$$

satisfies $\lim _{n \rightarrow \infty} g_{n}(\vartheta)=1$ for all $\vartheta \in \mathbb{R}^{d}$.

Lemma 3.5. For any $\vartheta=\left(\vartheta_{1}, \ldots, \vartheta_{d}\right) \in \mathbb{R}^{d}$ and $t>0$ we have

$$
\mathbb{E}\left[e^{i \vartheta \cdot X_{t}^{(n)}}\right]=\mathbb{E}\left[\left(\phi(1)-\phi\left(1-\frac{\cos \sqrt{\phi^{-1}\left(n^{-1}\right)} \vartheta_{1}+\ldots+\cos \sqrt{\phi^{-1}\left(n^{-1}\right)} \vartheta_{d}}{d}\right)^{N_{t}}\right] .\right.
$$

Proof. Since $\left(\xi_{k}\right)_{k \geqslant 1}$ is independent, identically distributed and independent of $N$, we get

$$
\mathbb{E}\left[e^{i \vartheta \cdot X_{t}^{(n)}}\right]=\mathbb{E}\left[\mathbb{E}\left[e^{i \sqrt{\phi^{-1}\left(n^{-1}\right) \vartheta \cdot \xi_{1}}}\right]^{N_{t}}\right]
$$

It is enough to note that

$$
\begin{aligned}
\mathbb{E}\left[e^{i \sqrt{\phi^{-1}\left(n^{-1}\right)} \vartheta \cdot \xi_{1}}\right] & =\sum_{m=1}^{\infty} \mathbb{E}\left[e^{\left.i \sqrt{\phi^{-1}\left(n^{-1}\right) \vartheta \cdot Z_{m}}\right]} \int_{(0, \infty)} \frac{t^{m}}{m !} e^{-t} \mu(d t)\right. \\
& =\sum_{m=1}^{\infty} \int_{(0, \infty)} \frac{\left(t \mathbb{E}\left[e^{i \sqrt{\phi^{-1}\left(n^{-1}\right)} \vartheta \cdot \zeta_{1}}\right]\right)^{m}}{m !} \mu(d t) \\
& =\int_{(0, \infty)}\left(e^{t \mathbb{E}\left[e^{i \sqrt{\phi^{-1}\left(n^{-1}\right)} \vartheta \cdot \zeta_{1}}\right]}-1\right) e^{-t} \mu(d t) \\
& =\phi(1)-\phi\left(1-\mathbb{E}\left[e^{i \sqrt{\phi^{-1}\left(n^{-1}\right)} \vartheta \cdot \zeta_{1}}\right]\right) .
\end{aligned}
$$

Now it is enough to note that

$$
\mathbb{E}\left[e^{i \sqrt{\phi^{-1}\left(n^{-1}\right)} \vartheta \cdot \zeta_{1}}\right]=\frac{\cos \sqrt{\phi^{-1}\left(n^{-1}\right)} \vartheta_{1}+\ldots+\cos \sqrt{\phi^{-1}\left(n^{-1}\right)} \vartheta_{d}}{d} .
$$


Proof of Proposition 1.3. To prove tightness we use Aldous' criterion (see [Ald78, Theorem $1]$ ), that is we show that for a sequence of bounded stopping times $\left(\tau_{n}\right)_{n \geqslant 1}$ (with respect to the natural filtration of $X^{(n)}$ for any $n \in \mathbb{N}$ ) and a sequence $\left(h_{n}\right)_{n \geqslant 1}$ of positive numbers converging to 0 the following holds

$$
Y_{n}:=X_{\tau_{n}+h_{n}}^{(n)}-X_{\tau_{n}}^{(n)} \text { converges to } 0 \text { in probability as } n \rightarrow \infty
$$

and, for any $t>0$ the following tightness of $\left(X_{t}^{(n)}\right)_{n \geqslant 1}$ : for any $\varepsilon>0$ there exists $K>0$ such that

$$
\limsup _{n \rightarrow \infty} \mathbb{P}\left(\left|X_{t}^{(n)}\right|>K\right)<\varepsilon .
$$

In order to prove (3.3), it is enough to prove convergence in distribution to 0. By Lemma 3.4 and Lemma 3.5 , for any $\vartheta \in \mathbb{R}^{d}$ we have

$$
\begin{aligned}
\lim _{n \rightarrow \infty} \mathbb{E}\left[e^{i \vartheta \cdot Y_{n}}\right] & =\lim _{n \rightarrow \infty} \mathbb{E}\left[e^{i \vartheta \cdot X_{h_{n}}^{(n)}}\right] \\
& =\lim _{n \rightarrow \infty} \mathbb{E}\left[\left(1-\phi\left(1-\frac{\cos \sqrt{\phi^{-1}\left(n^{-1}\right)} \vartheta_{1}+\ldots+\cos \sqrt{\phi^{-1}\left(n^{-1}\right)} \vartheta_{d}}{d}\right)\right)^{N_{n h_{n}}}\right] .
\end{aligned}
$$

Assume first that $N$ is the Poisson process with intensity 1. By using (3.5) and formula

$$
\mathbb{E}\left[\theta^{N_{t}}\right]=e^{t(\theta-1)}, \quad \theta, t>0
$$

we obtain

$$
\lim _{n \rightarrow \infty} \mathbb{E}\left[e^{i \vartheta \cdot Y_{n}}\right]=\lim _{n \rightarrow \infty} e^{-n h_{n} \phi\left(1-\frac{\cos \sqrt{\phi^{-1}\left(n^{-1}\right)} \vartheta_{1}+\ldots+\cos \sqrt{\phi^{-1}\left(n^{-1}\right)} \vartheta_{d}}{d}\right)}, \quad \vartheta \in \mathbb{R}^{d} .
$$

To evaluate the limit we use Lemma 3.1 (i) to get

$$
\begin{aligned}
0 & \leqslant n h_{n} \phi\left(1-\frac{\cos \sqrt{\phi^{-1}\left(n^{-1}\right)} \vartheta_{1}+\ldots+\cos \sqrt{\phi^{-1}\left(n^{-1}\right)} \vartheta_{d}}{d}\right) \\
& \leqslant n h_{n} \phi\left(\frac{\phi^{-1}\left(n^{-1}\right)|\vartheta|^{2}}{2 d}\right) \leqslant n h_{n}\left(\frac{|\vartheta|^{2}}{2 d} \vee 1\right) \phi\left(\phi^{-1}\left(n^{-1}\right)\right)=h_{n}\left(\frac{|\vartheta|^{2}}{2 d} \vee 1\right) .
\end{aligned}
$$

Then $\lim _{n \rightarrow \infty} \mathbb{E}\left[e^{i \vartheta \cdot Y_{n}}\right]=1$ and so convergence in distribution to 0 by continuity theorem. Further, by Lemma 3.3 it follows that, for any $\beta, t>0$ and $K \geqslant 1$,

$$
\begin{aligned}
\mathbb{P}\left(\left|X_{t}^{(n)}\right|>K\right) & =\mathbb{P}\left(\left|\sum_{k \leqslant N_{n t}} \xi_{k}\right|>\frac{K}{\sqrt{\left.\phi^{-1}\left(n^{-1}\right)\right)}}\right) \\
& =\sum_{m=1}^{\infty} \mathbb{P}\left(\left|\sum_{k \leqslant \frac{m}{n} n} \xi_{k}\right|>\frac{K}{\sqrt{\phi^{-1}\left(n^{-1}\right)}}\right) \mathbb{P}\left(N_{n t}=m\right) \\
& \leqslant \sum_{m=1}^{\infty} c_{0} \frac{m}{n}\left(K^{-2-\beta} \frac{\phi^{-1}\left(n^{-1}\right)}{\phi^{-1}\left(K^{-\beta} n^{-1}\right)}+K^{-\beta}\right) \frac{(n t)^{m}}{m !} e^{-n t} \\
& =c_{0}\left(K^{-2-\beta} \frac{\phi^{-1}\left(n^{-1}\right)}{\phi^{-1}\left(K^{-\beta} n^{-1}\right)}+K^{-\beta}\right) t \sum_{m=1}^{\infty} \frac{(n t)^{m-1}}{(m-1) !} e^{-n t} \\
& =c_{0} t\left(K^{-2-\beta} \frac{\phi^{-1}\left(n^{-1}\right)}{\phi^{-1}\left(K^{-\beta} n^{-1}\right)}+K^{-\beta}\right) .
\end{aligned}
$$


Therefore

$$
\limsup _{n \rightarrow \infty} \mathbb{P}\left(\left|X_{t}^{(n)}\right|>K\right) \leqslant c_{1} t\left(K^{-2-\beta+\beta \gamma}+K^{-\beta}\right)
$$

for some constant $c_{1}>0$ and so, by choosing $\beta>0$ small enough so that

$$
-2-\beta+\beta \gamma<0, \quad \text { i.e. } \quad \beta<\frac{2}{\gamma-1}
$$

and then $K \geqslant 1$ large enough, we get (3.4), finishing the proof of tightness in this case.

Let $N_{t}=\lfloor t\rfloor$. From (3.5) we obtain

$$
\begin{aligned}
\lim _{n \rightarrow \infty} \mathbb{E}\left[e^{i \vartheta \cdot Y_{n}}\right] & =\lim _{n \rightarrow \infty} e^{-\left\lfloor n h_{n}\right\rfloor \ln \left(1-\phi\left(1-\frac{\cos \sqrt{\phi^{-1}\left(n^{-1}\right)} \vartheta_{1}+\ldots+\cos \sqrt{\phi^{-1}\left(n^{-1}\right)} \vartheta_{d}}{d}\right)\right)} \\
& =\lim _{n \rightarrow \infty} e^{\left\lfloor n h_{n}\right\rfloor \phi\left(1-\frac{\cos \sqrt{\phi^{-1}\left(n^{-1}\right)} \vartheta_{1}+\ldots+\cos \sqrt{\phi^{-1}\left(n^{-1}\right)}}{d}\right)}=1
\end{aligned}
$$

by (3.7), showing the required convergence in distribution. Further, by Lemma 3.3, for any $t, \beta>0$ and $K \geqslant 1$

$$
\begin{aligned}
\limsup _{n \rightarrow \infty} \mathbb{P}\left(\left|X_{t}^{(n)}\right|>K\right) & =\limsup _{n \rightarrow \infty} \mathbb{P}\left(\left|\sum_{k \leqslant n t} \xi_{k}\right|>\frac{K}{\sqrt{\phi^{-1}\left(n^{-1}\right)}}\right) \\
& \leqslant c_{0} t \limsup _{n \rightarrow \infty}\left(K^{-2-\beta} \frac{\phi^{-1}\left(n^{-1}\right)}{\phi^{-1}\left(K^{-\beta} n^{-1}\right)}+K^{-\beta}\right) \leqslant c_{1} t\left(K^{-2-\beta+\beta \gamma}+K^{-\beta}\right) .
\end{aligned}
$$

Choosing $\beta>0$ as in (3.8) we may choose $K \geqslant 1$ large enough so that (3.4) holds.

\section{WEAK CONVERGENCE}

We start with a few auxilliary results.

Lemma 4.1. Let $\left(a_{n}\right)_{n \geqslant 1}$ and $\left(b_{n}\right)_{n \geqslant 1}$ be sequences of non-negative numbers such that $\lim _{n \rightarrow \infty} a_{n}=a \in(0, \infty), \lim _{n \rightarrow \infty} b_{n}=0$ and let $f:(0, \infty) \rightarrow(0, \infty)$ be a monotone function that varies regularly at 0 with index $\rho \in \mathbb{R}$. Then

$$
\lim _{n \rightarrow \infty} \frac{f\left(a_{n} b_{n}\right)}{f\left(b_{n}\right)}=a^{\rho} .
$$

Proof. Assume that $f$ is non-decreasing. For any $0<\varepsilon<a$ and $n \in \mathbb{N}$ large enough we have

$$
\frac{f\left((a-\varepsilon) b_{n}\right)}{f\left(b_{n}\right)} \leqslant \frac{f\left(a_{n} b_{n}\right)}{f\left(b_{n}\right)} \leqslant \frac{f\left((a+\varepsilon) b_{n}\right)}{f\left(b_{n}\right)} .
$$

This implies

$$
(a-\varepsilon)^{\rho} \leqslant \liminf _{n \rightarrow \infty} \frac{f\left(a_{n} b_{n}\right)}{f\left(b_{n}\right)} \leqslant \limsup _{n \rightarrow \infty} \frac{f\left(a_{n} b_{n}\right)}{f\left(b_{n}\right)} \leqslant(a+\varepsilon)^{\rho} .
$$

Letting $\varepsilon \rightarrow 0$ we obtain

$$
\lim _{n \rightarrow \infty} \frac{f\left(a_{n} b_{n}\right)}{f\left(b_{n}\right)}=a^{\rho} .
$$

If $f$ is non-increasing, the proof is similar. 
Lemma 4.2. Let $\phi:(0, \infty) \rightarrow(0, \infty)$ be a Bernstein function.

(i) If $\phi$ varies regularly at 0 with index $\rho \in \mathbb{R}$, then $\rho \in[0,1]$.

(ii) Let $\left(a_{n}\right)_{n \geqslant 1}$ be a sequence of positive numbers such that $a_{n} \leqslant 1$ for all $n \in \mathbb{N}$ and $\lim _{n \rightarrow \infty} a_{n}=1$. Then

$$
\lim _{n \rightarrow \infty} \frac{\phi\left(a_{n} x\right)}{\phi(x)}=1 \text { uniformly in } x>0 .
$$

Proof. (i) Let $x \geqslant 1$. By Lemma 3.1 (ii), it follows that

$$
x \geqslant \lim _{\lambda \downarrow 0} \frac{\phi(\lambda x)}{\phi(\lambda)}=x^{\rho},
$$

hence $\rho \leqslant 1$. On the other hand, since $\phi$ is non-decreasing,

$$
1 \leqslant \lim _{\lambda \downarrow 0} \frac{\phi(\lambda x)}{\phi(\lambda)}=x^{\rho}
$$

and so $\rho \geqslant 0$.

(ii) By Lemma 3.1 (ii), for any $x>0, \phi(x)=\phi\left(a_{n} x a_{n}^{-1}\right) \leqslant a_{n}^{-1} \phi\left(a_{n} x\right)$ and so

$$
a_{n} \leqslant \frac{\phi\left(a_{n} x\right)}{\phi(x)} \leqslant 1
$$

yielding the claim.

The following sufficient result for regular variation is taken from [Fel71].

Lemma 4.3. Let $\left(\lambda_{n}\right)_{n \geqslant 1}$ and $\left(a_{n}\right)_{n \geqslant 1}$ be sequences of positive numbers such that

$$
\lim _{n \rightarrow \infty} \frac{a_{n}}{a_{n+1}}=1 \quad \text { and } \quad \lim _{n \rightarrow \infty} \lambda_{n}=0 .
$$

If $f:(0, \infty) \rightarrow(0, \infty)$ is monotone,

$$
g(y)=\lim _{n \rightarrow \infty} a_{n} f\left(\lambda_{n} y\right) \in[0, \infty]
$$

exists on a dense subset of $(0, \infty)$ and it is finite and positive on some interval, then $f$ varies regularly at 0 with index $\alpha \in \mathbb{R}$.

Proof. See [Fel71, Lemma VIII.8.3].

Proof of Theorem 1.4. Assume that $X^{(n)}$ converges to $X=\left(X_{t}\right)_{t \geqslant 0}$ in $D\left([0, \infty), \mathbb{R}^{d}\right)$. Then $X_{t}^{(n)}$ converges in distribution to $X_{t}$ for any $t>0$ and, by Lemma 3.5 the following limit exists

$$
\lim _{n \rightarrow \infty} \mathbb{E}\left[e^{i X_{t}^{(n)} \cdot \vartheta}\right]=\lim _{n \rightarrow \infty} \mathbb{E}\left[\left(1-\phi\left(1-\frac{\cos \sqrt{\phi^{-1}\left(n^{-1}\right)} \vartheta_{1}+\ldots+\cos \sqrt{\phi^{-1}\left(n^{-1}\right)} \vartheta_{d}}{d}\right)\right)^{N_{n t}}\right]
$$

for any $\vartheta=\left(\vartheta_{1}, \ldots, \vartheta_{d}\right) \in \mathbb{R}^{d}$. If $\left(N_{t}\right)_{t \geqslant 0}$ is the Poisson process with intensity 1 by $(3.6)$ and (4.1) the following limit exists

$$
\lim _{n \rightarrow \infty} \mathbb{E}\left[e^{i X_{t}^{(n)} \cdot \vartheta}\right]=\lim _{n \rightarrow \infty} e^{-n t \phi\left(1-\frac{\cos \sqrt{\phi^{-1}\left(n^{-1}\right)} \vartheta_{1}+\ldots+\cos \sqrt{\phi^{-1}\left(n^{-1}\right)} \vartheta_{d}}{d}\right)} .
$$


For $N_{t}=\lfloor t\rfloor$, we see that the following limit exists

$$
\begin{aligned}
\lim _{n \rightarrow \infty} \mathbb{E}\left[e^{i X_{t}^{(n)} \cdot \vartheta}\right] & =\lim _{n \rightarrow \infty} e^{-\lfloor n t\rfloor \log \left(1-\phi\left(1-\frac{\cos \sqrt{\phi^{-1}\left(n^{-1}\right) \vartheta_{1}}+\ldots+\cos \sqrt{\phi^{-1}\left(n^{-1}\right)} \vartheta_{d}}{d}\right)\right)} \\
& =\lim _{n \rightarrow \infty} e^{-\operatorname{tn\phi }\left(1-\frac{\cos \sqrt{\phi^{-1}\left(n^{-1}\right)} \vartheta_{1}+\ldots+\cos \sqrt{\phi^{-1}\left(n^{-1}\right)} \vartheta_{d}}{d}\right)} .
\end{aligned}
$$

Therefore, in both cases

$$
\lim _{n \rightarrow \infty} n \phi\left(1-\frac{\cos \sqrt{\phi^{-1}\left(n^{-1}\right)} \vartheta_{1}+\ldots+\cos \sqrt{\phi^{-1}\left(n^{-1}\right)} \vartheta_{d}}{d}\right)
$$

exists.

Define

$$
a_{n}=\frac{1-\frac{\cos \sqrt{\phi^{-1}\left(n^{-1}\right)} \vartheta_{1}+\ldots+\cos \sqrt{\phi^{-1}\left(n^{-1}\right)} \vartheta_{d}}{d}}{\frac{\phi^{-1}\left(n^{-1}\right)|\vartheta|^{2}}{2 d}} \text { and } \quad b_{n}=\phi^{-1}\left(n^{-1}\right) \frac{|\vartheta|^{2}}{2 d} .
$$

Since $a_{n} \leqslant 1$ for all $n \in \mathbb{N}, \lim _{n \rightarrow \infty} a_{n}=1$ and $\lim _{n \rightarrow \infty} b_{n}=0$, Lemma 4.2 implies that

$$
\lim _{n \rightarrow \infty} \frac{\phi\left(1-\frac{\cos \sqrt{\phi^{-1}\left(n^{-1}\right)} \vartheta_{1}+\ldots+\cos \sqrt{\phi^{-1}\left(n^{-1}\right)} \vartheta_{d}}{d}\right)}{\phi\left(\phi^{-1}\left(n^{-1}\right) \frac{|\vartheta|^{2}}{2 d}\right)}=\lim _{n \rightarrow \infty} \frac{\phi\left(a_{n} b_{n}\right)}{\phi\left(b_{n}\right)}=1 ;
$$

hence, in both cases, the limit

$$
\lim _{n \rightarrow \infty} n \phi\left(\phi^{-1}\left(n^{-1}\right) \frac{|\vartheta|^{2}}{2 d}\right)=\lim _{n \rightarrow \infty} n \phi\left(1-\frac{\cos \sqrt{\phi^{-1}\left(n^{-1}\right)} \vartheta_{1}+\ldots+\cos \sqrt{\phi^{-1}\left(n^{-1}\right)} \vartheta_{d}}{d}\right)
$$

exists. By Lemma 4.3 (with $\lambda_{n}=\phi^{-1}\left(n^{-1}\right)$ and $a_{n}=n$ ) we conclude that $\phi$ varies regularly at 0 with index $\alpha \in \mathbb{R}$. Lemma 4.1 ensures that $0 \leqslant \alpha \leqslant 1$. Note that (4.1), (4.5) and (4.2) (or (4.3)) yield

$$
\mathbb{E}\left[e^{i \vartheta \cdot X_{t}}\right]=\lim _{n \rightarrow \infty} \mathbb{E}\left[e^{i \vartheta \cdot X_{t}^{(n)}}\right]=\lim _{n \rightarrow \infty} e^{-t n \phi\left(\phi^{-1}\left(n^{-1}\right) \frac{|\vartheta|^{2}}{2 d}\right)}=\lim _{n \rightarrow \infty} e^{-t \frac{\phi\left(\phi^{-1}\left(n^{-1}\right) \frac{|\vartheta|^{2}}{2 d}\right)}{\phi\left(\phi^{-1}\left(n^{-1}\right)\right)}} .
$$

Hence, if $\alpha \in(0,1]$

$$
\mathbb{E}\left[e^{i \vartheta \cdot X_{t}}\right]=e^{-t(2 d)^{-\alpha}|\vartheta|^{2 \alpha}}
$$

and so $X=(2 d)^{-\alpha} W^{(2 \alpha)}$ is a multiple of a rotationally invariant $2 \alpha$-stable process. On the other hand, if $\alpha=0$ we get

$$
\mathbb{E}\left[e^{i \vartheta \cdot X_{t}}\right]=e^{-t}
$$

which is impossible, since for $\vartheta \rightarrow 0$ we get a contradiction: $1=e^{-t}$ for all $t \geqslant 0$.

Assume that $\phi$ varies regularly at 0 with index $\alpha \in(0,1]$. By Lemma 3.1 (iii), $\phi^{-1}$ varies at 0 with index $1 / \alpha$ and so

$$
\limsup _{\lambda \downarrow 0} \frac{\phi^{-1}(\lambda x)}{\phi^{-1}(\lambda)} \leqslant x^{1 / \alpha}, \quad x \geqslant 1 .
$$

Note that we can always replace $1 / \alpha$ in the last display by any $\gamma>1 / \alpha \geqslant 1$. Hence we may use Proposition 1.3 to obtain tightness of the sequence $\left(X^{(n)}\right)_{n \geqslant 1}$ in $D\left[(0, \infty), \mathbb{R}^{d}\right)$. To prove convergence, it is enough to prove convergence of finite-dimensional distributions 
(see [Kal02, Theorem 16.10]). By Lemma 3.4, for any $0 \leqslant s<t$ (with $\tau_{n}=s$ i $h_{n}=t-s$ ) we get

$$
\lim _{n \rightarrow \infty} \mathbb{E}\left[e^{i \vartheta \cdot\left(X_{t}^{(n)}-X_{s}^{(n)}\right)}\right]=\lim _{n \rightarrow \infty} \mathbb{E}\left[e^{i \vartheta \cdot X_{t-s}^{(n)}}\right]
$$

Hence, by (4.6),

$$
\lim _{n \rightarrow \infty} \mathbb{E}\left[e^{i \vartheta \cdot\left(X_{t}^{(n)}-X_{s}^{(n)}\right)}\right]=e^{-(t-s)(2 d)^{-\alpha}|\vartheta|^{2 \alpha}} .
$$

Let $j \in \mathbb{N}, 0=t_{0} \leqslant t_{1}<t_{2}<\ldots<t_{j}$ and $\vartheta^{(1)}, \vartheta^{(2)}, \ldots, \vartheta^{(j)} \in \mathbb{R}^{d}$. Using independent increments property of Lévy processes (if $N$ is Poisson process) or independence (if $N_{t}=$ $\lfloor t\rfloor)$ and (4.7) we get

$$
\begin{aligned}
\lim _{n \rightarrow \infty} \mathbb{E} \exp \left\{i \sum_{k=1}^{j} \vartheta^{(k)} \cdot X_{t_{k}}^{(n)}\right\} & =\lim _{n \rightarrow \infty} \mathbb{E} \exp \left\{i \sum_{k=1}^{j} \sum_{l=1}^{k} \vartheta^{(k)} \cdot\left(X_{t_{l}}^{(n)}-X_{t_{l-1}}^{(n)}\right)\right\} \\
& =\lim _{n \rightarrow \infty} \mathbb{E} \exp \left\{i \sum_{l=1}^{j} \sum_{k=l}^{j} \vartheta^{(k)} \cdot\left(X_{t_{l}}^{(n)}-X_{t_{l-1}}^{(n)}\right)\right\} \\
& =\prod_{l=1}^{j} \lim _{n \rightarrow \infty} \mathbb{E} \exp \left\{i \sum_{k=l}^{j} \vartheta^{(k)} \cdot\left(X_{t_{l}}^{(n)}-X_{t_{l-1}}^{(n)}\right)\right\} \\
& =\prod_{l=1}^{j} \exp \left\{-\left(t_{l}-t_{l-1}\right)(2 d)^{-\alpha}\left|\sum_{k=l}^{j} \vartheta^{(k)}\right|^{2 \alpha}\right\} \\
& =\mathbb{E} \exp \left\{i \sum_{k=1}^{j} \vartheta^{(k)} \cdot X_{t_{k}}\right\}
\end{aligned}
$$

where the last line is obtained by using independent increments property of a Lévy process $X=(2 d)^{-\alpha} W^{(\alpha)}$. Now it is enough to apply continuity theorem for characteristic functions to obtain convergence in distrubution of $\left(X_{t_{1}}^{(n)}, \ldots, X_{t_{j}}^{(n)}\right)$ to $\left(X_{t_{1}}, \ldots, X_{t_{j}}\right)$.

\section{REFERENCES}

[Ald78] D. Aldous, Stopping times and tightness, Ann. Probab. 6 (1978), no. 2, 335-340.

[Ber96] J. Bertoin, Lévy processes, Cambridge University Press, Cambridge, 1996.

[BGT87] N. H. Bingham, C. M. Goldie, and J. L. Teugels, Regular variation, Cambridge University Press, Cambridge, 1987.

[BSC12] A. Bendikov and L. Saloff-Coste, Random walks on groups and discrete subordination, Math. Nachr. 285 (2012), 580-605.

[EKM03] P. Embrechts, C. Klüpperberg, and T. Mikosch, Modelling extremal events, Springer, Berlin, 2003.

[Fel71] W. Feller, An introduction to probability theory and its applications, John Wiley and Sons, New York, 1971.

[Gut09] A. Gut, Stopped random walks, Springer, Berlin, 2009.

[JS03] J. Jacod and A. N. Shiryaev, Limit theorems for stochastic processes, Springer, Berlin, 2003.

[Kal02] O. Kalenberg, Foundations of modern probability, Springer, Berlin, 2002.

[Sat99] K.-I. Sato, Lévy processes and infinitely divisible distributions, Cambridge University Press, Cambridge, 1999. 
[SSV12] R. L. Schilling, R. Song, and Z. Vondraček, Bernstein functions: theory and applications, Walter de Gruyter, Berlin, 2012.

University of Zagreb, Department of Mathematics, BijeničKa Cesta 30, 10000 Zagreb, Croatia

E-mail address: amimica@math.hr 